\title{
The Effects of Interferential Stimulation on Pain and Motion after Shoulder Surgery: A Prospective Randomized Study
}

\author{
Vishal Mehta \\ Fox Valley Orthopaedic Institute, USA \\ Email: vmehta44@gmail.com \\ Received 16 April 2015; accepted 23 October 2015; published 26 October 2015 \\ Copyright (C) 2015 by author and Scientific Research Publishing Inc. \\ This work is licensed under the Creative Commons Attribution International License (CC BY). \\ http://creativecommons.org/licenses/by/4.0/

(c) (i) Open Access

\section{Abstract}

Purpose: The purpose of this study is to determine the effects of interferential stimulation on pain and motion after shoulder surgery. Study Design: Randomized Controlled Trial. Methods: 102 patients undergoing arthroscopic rotator cuff repair or labral repair were prospectively randomized to receive either an interferential stimulation unit immediately after surgery (STIM) or not to receive the unit (NO-STIM). Each patient was measured for the range of motion (ROM) in forward flexion (FF), abduction (ABD), internal rotation (IR) and external rotation (ER). These measurements were taken prior to surgery and at the 6 weeks post-operative time point. Visual Analogue Scale (VAS) scores ( 0 - 10) and the amount of narcotic taken (standardized to $10 \mathrm{mg}$ equivalents of hydrocodone) were measured and compared between the two groups at 2 days and 4 weeks post-operatively. 51 patients were excluded due to failure to document VAS scores and medication usage, lack of follow-up at the 6 weeks post-operative time point or hospitalization after surgery. This left 27 patients in the STIM group and 24 patients in the NO-STIM group. Results: No difference was found in the amount of narcotic taken after 2 days (STIM $14.37 \pm 1.02$, NO-STIM $15.88 \pm$ $1.22, p=0.34$ ) or after 4 weeks (STIM 45.32 \pm 4.36 , NO-STIM $48.96 \pm 5.50, p=0.60$ ). No difference in mean VAS scores were found at 2 days (STIM $5.56 \pm 0.53$, NO-STIM $4.63 \pm 0.57, p=0.24$ ) or 4 weeks (STIM $1.68 \pm 0.39$, NO-STIM $1.38 \pm 0.34, p=0.57$ ). At 6 weeks post-operatively, a difference was found in ER (STIM 62.5 \pm 4.09 , NO-STIM 50.4 $\pm 4.09, p=0.04$ ), and no differences found in FF (STIM 134.2 \pm 5.29, NO-STIM 133.3 \pm 4.85 , $\mathrm{p}=0.60$ ), ABD (STIM 124.8 \pm 6.22 , NO-STIM $119.6 \pm 5.42$, $p=0.53$ ) or IR (STIM $60.0 \pm 3.69$, NO-STIM $55.9 \pm 3.03, p=0.39$ ). A post-hoc power analysis performed using an á $=0.05$ revealed the study to be $90 \%$ powered to identify a difference of one narcotic equivalent between groups and $90 \%$ powered to identify a 0.3 difference in VAS scores. Conclusions: The use of an interferential stimulator in the immediate post-operative period had no effect on pain or narcotic usage following arthroscopic rotator cuff repair or labral repair. External rotation was significantly greater in the interferential stimulator group while no differences were found in other motion parameters. 


\section{Keywords}

\section{Shoulder, Arthroscopy, Pain, Interferential Stimulation, Motion}

\section{Introduction}

The use of Interferential Stimulation (IFS) after orthopedic procedures has grown in popularity in recent years. Advocates of IFS believe that it decreases post-operative pain and may also improve post-operative motion. It has been used extensively after total knee arthroplasty as well as after shoulder and knee stabilization procedures.

Interferential stimulation, also known as interferential current therapy (IFC), became increasingly popular as a therapeutic modality in the 1950s [1]. The use of electrical currents in different forms has actually taken place for hundreds of years [2]. Over the past century, the use of electrical stimulation as a therapeutic modality has evolved into the modern day version of interferential stimulation. In its most common application, IFS uses alternating currents at a frequency of up to $4150 \mathrm{~Hz}$. Two separate pairs of electrodes are positioned diagonally across from each other in order to produce an "interference" where these currents intersect. It is postulated that the mode of action of IFS is to depolarize peripheral sensory and motor nerve fibers [3]-[5].

Interferential stimulation has been used in a variety of clinical settings, from managing back pain and other musculoskeletal disorders to the hyperreflexia associated with multiple sclerosis. It has also been used on chronic wounds and non-unions and even to improve urinary dysfunction. Data on its efficacy in knee and shoulder surgery are limited. To date, there is no published research evaluating the effects of an IFS unit during the immediate post-operative period following arthroscopic shoulder surgery. This study seeks to fill the void in the literature and determine the effects of a home IFS unit on post-operative pain, range-of-motion, and the narcotics consumption.

\section{Method}

102 consecutive patients undergoing arthroscopic rotator cuff repair or labral repair were prospectively randomized to receive either an interferential stimulation unit (TL-1000, TheraLabs, Naperville, IL) immediately after surgery (STIM) or not to receive the unit (NO-STIM). Patient demographics were comparable in both groups (Table 1). Approval from the Internal Review Board was obtained prior to the start of this study.

Patients in the STIM group were given a TL-1000 unit for home use (Figure 1). Prior to surgery, a representative from TheraLabs visited each patient to provide specific instructions on the stimulator unit, including proper electrode placement, frequency of use, and settings. A detailed instructional sheet was also given to each patient. Initial amplitude settings were established based on the patient's tolerance. The electrodes were applied in the operating room under sterile conditions, prior to the application of a surgical dressing. The stimulator units were turned on in the recovery room and set to $75 \%$ of the previously determined patient-specific tolerance. The usual initial amplitude settings were from $40-70 \mathrm{~Hz}$. Patients were instructed to use the stimulator 3 times per day for 20 minutes at a time, allowing at least 4 hours in between sessions. Once discharged home, the patient was instructed to adjust the amplitude as high as could be tolerated without increasing pain and without causing muscle spasms.

Each patient was measured for range of motion (ROM) in forward flexion (FF), abduction (ABD), internal rotation (IR) and external rotation (ER). These measurements were taken prior to surgery and at the 6 weeks

\section{Table 1. Demographic data.}

\begin{tabular}{cccc}
\hline & STIM & NO-STIM & p value \\
\hline Gender & & & 0.75 \\
Men & $20(74 \%)$ & $19(79 \%)$ & \\
Women & $7(26 \%)$ & $5(21 \%)$ & 0.69 \\
Age & $51.27(18-76)$ & $49.52(19-75)$ & \\
\hline
\end{tabular}




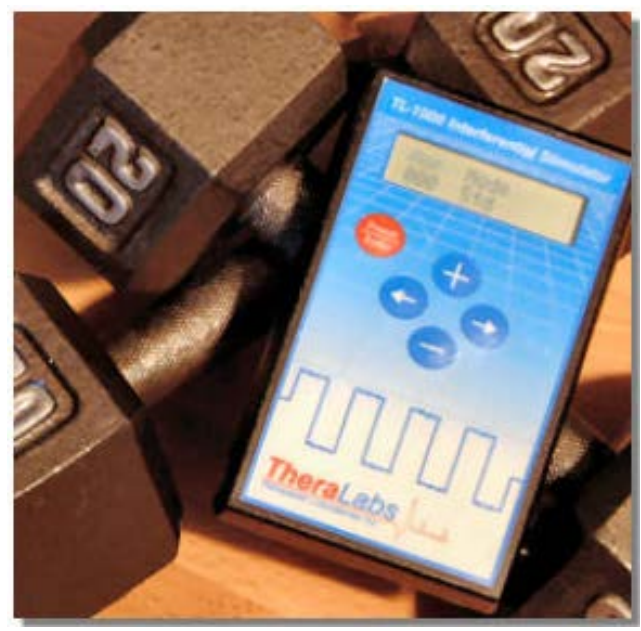

Figure 1. Photograph of interferential stimulation device used in this study.

post-operative time point and represent passive range of motion taken with the scapula stabilized. Visual Analogue Scale (VAS) scores $(0-10)$ and the amount of narcotic taken (standardized to $10 \mathrm{mg}$ equivalents of hydrocodone) were measured and compared between the two groups at 2 days and 4 weeks post-operatively. 51 patients were excluded due to failure to document VAS scores and medication usage, lack of follow-up at the 6 weeks post-operative time point or hospitalization after surgery. This left 27 patients in the STIM group and 24 patients in the NO-STIM group.

\section{Surgical Technique}

\subsection{Rotator Cuff Repair}

All rotator cuff repairs were performed using an arthroscopic technique. No open or mini-open repairs were included in this study. There was a mix of single row and double row repairs performed with most double-row repairs using a trans-osseous equivalent technique.

\subsection{Labral Repair}

All labral repairs were performed arthroscopically and 1 - 5 anchors were used for labral repair. Knotted bioabsorbable anchors, Bio-Suture Tack (Arthrex, Naples, FL), were used for all repairs. Rotator interval closure was not routinely performed.

\subsection{Rehabilitation}

All patients were placed in a sling for 6 weeks post-operatively and underwent formal physical therapy. Rotator cuff repairs usually began physical therapy at the 2 - 3 weeks point but this was individualized for each patient. Active range of motion was not permitted until the 6 week point. Labral repairs began physical therapy at the 1 2 week point but therapy was postponed for high risk cases. Patients who underwent Bankart repairs were not allowed to reach a combined 90 degrees of abduction and external rotation until the 6 week point.

\subsection{Statistical Methods}

A student t-test was used to compare VAS, ROM and narcotic usage between the two groups with a p value set at $<0.05$. As most of the outcomes did not reach statistical significance, a post-hoc power analysis was performed using an alpha value of 0.05 .

\section{Results}

No difference was found in the amount of narcotic taken after 2 days (STIM $14.37 \pm 1.02$, NO-STIM $15.88 \pm$ 
1.22, $\mathrm{p}=0.34$ ) (Figure 2) or after 4 weeks (STIM $45.32 \pm 4.36$, NO-STIM $48.96 \pm 5.50, \mathrm{p}=0.6$ ) (Figure 3). No difference in mean VAS scores was found at 2 days (STIM $5.56 \pm 0.53$, NO-STIM $4.63 \pm 0.57, \mathrm{p}=0.24$ ) (Figure 4) or 4 weeks (STIM $1.68 \pm 0.39$, NO-STIM $1.38 \pm 0.34, \mathrm{p}=0.57$ ) (Figure 5). At 6 weeks post-operatively, a difference was found in ER (STIM $62.5 \pm 4.09$, NO-STIM $50.36 \pm 4.09, \mathrm{p}=0.041$ ), and no differences found in FF (STIM $134.2 \pm 5.29$, NO-STIM $133.3 \pm 4.85$, p = 0.60), ABD (STIM $124.8 \pm 6.22$, NO-STIM $119.6 \pm 5.42$, p = 0.53.) or IR (STIM $60.00 \pm 3.69$, NO-STIM $55.92 \pm 3.03$, p = 0.39). A post-hoc power analysis performed using an $\alpha=0.05$ revealed the study to be $90 \%$ powered to identify a difference of one narcotic equivalent between groups and 90\% powered to identify a 0.3 difference in VAS scores (Figures 2-5).

Pain Medication Taken After 2 Days

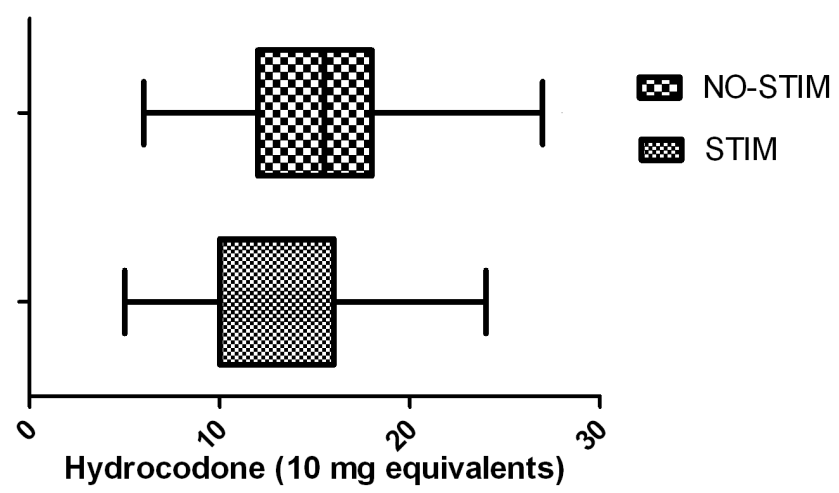

Figure 2. Amount of pain medication taken after 2 days.

\section{Pain Medication Taken After 4 Weeks}

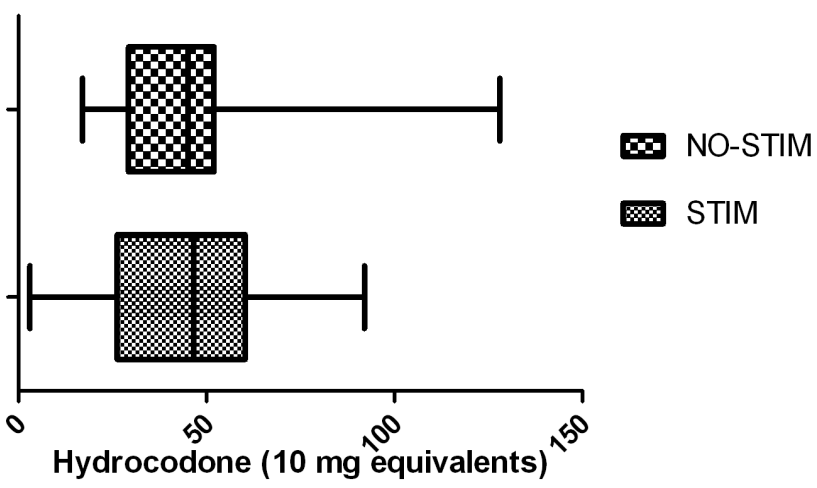

Figure 3. Amount of pain medication taken after 4 weeks.

Mean VAS at 2 Days After Surgery

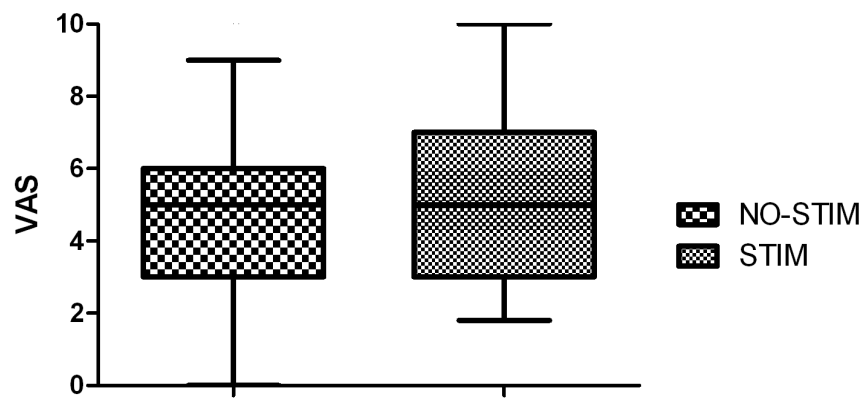

Figure 4. Visual Analogue Pain Scale after 2 days. 


\section{Mean VAS at 4 Weeks After Surgery}

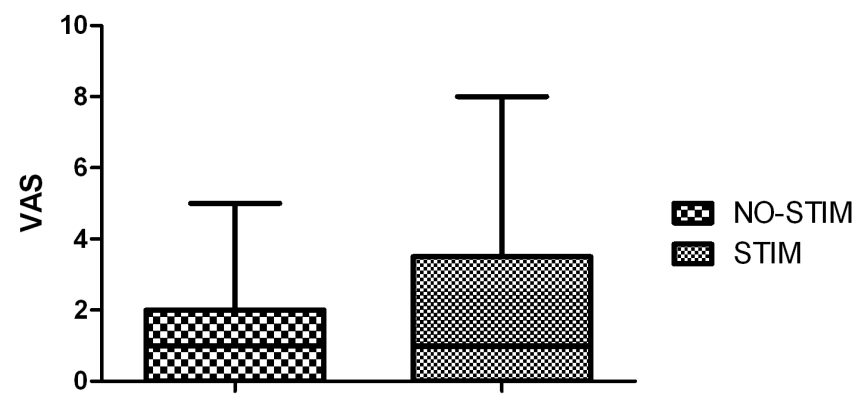

Figure 5. Visual analogue pain scale after 4 weeks.

\section{Discussion}

The use of interferential stimulation in the immediate post-operative period has been advocated as an effective technique to reduce pain and improve motion. While IFS has been studied in other areas, its' efficacy following orthopedic procedures has not been adequately studied.

The ability of IFS to have an effect on pain pathways has been clearly documented. Johnson et al. demonstrated that IFS could decrease ischemic pain that was induced in young healthy volunteers [6]. Similar studies have shown the beneficial effects of IFS on induced pain in controlled human studies [7]-[9]. Noble et al. also demonstrated the ability of IFS to improve blood flow in humans [10]. Similar positive effects of IFS have been demonstrated on back pain and in psoriatic arthritis [3] [4] [11]. Almeida et al. studied the effects of IFS on fibromyalgia and pain and found a beneficial effect with the use of IFS in the subjects involved [12].

While there are many studies demonstrating the efficacy of IFS on different ailments, there are also studies suggesting that IFS has little or no effect on pain [13]-[15]. Minder et al. demonstrated that IFS had no effect on induced muscle soreness [5]. In a randomized study of the effects of IFS on what the authors describe as "softtissue shoulder disorders", Van Der Heijden et al. demonstrated no improvement in pain with the use of IFS [16]. Jarit et al. performed a randomized, controlled, double-blinded study on the use of IFS following Anterior Cruciate Ligament (ACL) reconstruction [17]. In this well designed and executed study, they demonstrated decreased pain, improved motion and decreased edema with the use of IFS.

To the best of our knowledge, this is the first study to attempt to determine the efficacy of IFS on post-operative pain and motion following arthroscopic shoulder surgery. Our study does not reproduce the beneficial effects the Jarit et al. found in their study of the knee. The reason for this is unclear. While, this is not an extremely large study, post-hoc power analysis reveals that it is sufficiently powered. Other limitations to this study include the fact that it is not placebo controlled. The initial study protocol was written with a sham IFS device to serve as a placebo group but this was rejected by the Internal Review Board (IRB). The IRB believed patients would be able to tell that they were not receiving therapeutic levels of IFS and that this could, in fact, create a negative placebo effect. For this reason a placebo was not used. Certainly, it could be possible that other "doses" of IFS or different timings may be more optimal than what was studied here.

\section{Conclusion}

In conclusion, we demonstrate no decrease in pain or narcotic consumption with the use of IFS after arthroscopic shoulder surgery. The use of IFS may lead to improvements in early post-operative motion. Care should be taken not to generalize this study to the use of IFS in general. With many previous studies demonstrating some efficacy with the use of IFS, there are likely some prudent clinical applications. However, we do not believe one of those applications to be the reduction of pain after arthroscopic shoulder surgery.

\section{References}

[1] Ward, A.R. (2009) Electrical Stimulation Using Kilohertz-Frequency Alternating Current. Physical Therapy, 89, 181-190. http://dx.doi.org/10.2522/ptj.20080060

[2] Robinson, A.J. and Snyder-Mackler, L. (2008) Clinical Electrophysiology: Electrotherapy and Electrophysiologic 
Testing. 3rd Edition, Lippincott Williams \& Wilkins, Philadelphia.

[3] Walker, U.A., et al. (2006) Analgesic and Disease Modifying Effects of Interferential Current in Psoriatic Arthritis. Rheumatology International, 26, 904-907. http://dx.doi.org/10.1007/s00296-006-0102-y

[4] Zambito, A., et al. (2006) Interferential and Horizontal Therapies in Chronic Low Back Pain: A Randomized, Double Blind, Clinical Study. Clinical and Experimental Rheumatology, 24, 534-539.

[5] Minder, P.M., et al. (2002) Interferential Therapy: Lack of Effect upon Experimentally Induced Delayed Onset Muscle Soreness. Clinical Physiology and Functional Imaging, 22, 339-347. http://dx.doi.org/10.1046/j.1475-097X.2002.00441.x

[6] Johnson, M.I. and Tabasam, G. (2002) A Single-Blind Placebo-Controlled Investigation into the Analgesic Effects of Interferential Currents on Experimentally Induced Ischaemic Pain in Healthy Subjects. Clinical Physiology and Functional Imaging, 22, 187-196. http://dx.doi.org/10.1046/j.1475-097X.2002.00416.X

[7] Cheing, G.L. and Hui-Chan, C.W. (2003) Analgesic Effects of Transcutaneous Electrical Nerve Stimulation and Interferential Currents on Heat Pain in Healthy Subjects. Journal of Rehabilitation Medicine, 35, 15-19. http://dx.doi.org/10.1080/16501970306101

[8] Cramp, F.L., et al. (2000) A Controlled Study on the Effects of Transcutaneous Electrical Nerve Stimulation and Interferential Therapy upon the RIII Nociceptive and H-Reflexes in Humans. Archives of Physical Medicine and Rehabilitation, 81, 324-333. http://dx.doi.org/10.1016/S0003-9993(00)90079-0

[9] Johnson, M.I. and Tabasam, G. (2003) An Investigation into the Analgesic Effects of Interferential Currents and Transcutaneous Electrical Nerve Stimulation on Experimentally Induced Ischemic Pain in Otherwise Pain-Free Volunteers. Physical Therapy, 83, 208-223.

[10] Noble, J.G., et al. (2000) The Effect of Interferential Therapy upon Cutaneous Blood Flow in Humans. Clinical Physiology, 20, 2-7. http://dx.doi.org/10.1046/j.1365-2281.2000.00207.x

[11] Hurley, D.A., et al. (2001) Interferential Therapy Electrode Placement Technique in Acute Low Back Pain: A Preliminary Investigation. Archives of Physical Medicine and Rehabilitation, 82, 485-493. http://dx.doi.org/10.1053/apmr.2001.21934

[12] Almeida, T.F., et al. (2003) The Effect of Combined Therapy (Ultrasound and Interferential Current) on Pain and Sleep in Fibromyalgia. Pain, 104, 665-672. http://dx.doi.org/10.1016/S0304-3959(03)00139-8

[13] Johnson, M.I. and Tabasam, G. (2003) An Investigation into the Analgesic Effects of Different Frequencies of the Amplitude-Modulated Wave of Interferential Current Therapy on Cold-Induced Pain in Normal Subjects. Archives of Physical Medicine and Rehabilitation, 84, 1387-1394. http://dx.doi.org/10.1016/S0003-9993(03)00151-5

[14] Johnson, M.I. and Tabasam, G. (2003) A Single-Blind Investigation into the Hypoalgesic Effects of Different Swing Patterns of Interferential Currents on Cold-Induced Pain in Healthy Volunteers. Archives of Physical Medicine and Rehabilitation, 84, 350-357. http://dx.doi.org/10.1053/apmr.2003.50005

[15] Taylor, K., et al. (1987) Effects of Interferential Current Stimulation for Treatment of Subjects with Recurrent Jaw Pain. Physical Therapy, 67, 346-350.

[16] Van Der Heijden, G.J., et al. (1999) No Effect of Bipolar Interferential Electrotherapy and Pulsed Ultrasound for Soft Tissue Shoulder Disorders: A Randomised Controlled Trial. Annals of the Rheumatic Diseases, 58, 530-540. http://dx.doi.org/10.1136/ard.58.9.530

[17] Jarit, G.J., et al. (2003) The Effects of Home Interferential Therapy on Post-Operative Pain, Edema, and Range of Motion of the Knee. Clinical Journal of Sport Medicine, 13, 16-20. http://dx.doi.org/10.1097/00042752-200301000-00004 